Technical note

\title{
Pre-hybridisation: An efficient way of suppressing endogenous biotin-binding activity inherent to biotin-streptavidin detection system
}

\author{
Raju Ahmed ${ }^{\mathrm{a}, *}$, Emma Spikings ${ }^{\mathrm{a}}$, Shaobo Zhou ${ }^{\mathrm{a}}$, Andrew Thompsett ${ }^{\mathrm{b}}$, Tiantian Zhang $^{\mathrm{c}}$ \\ a Institute of Biomedical and Environmental Sciences and Technology (iBEST), University of Bedfordshire, 250 Butterfield, Great Marlings, Luton, \\ Bedfordshire LU2 8DL, UK \\ ${ }^{\mathrm{b}}$ School of Health, Sport and Bioscience, University of East London, Stratford Campus, London E15 4LZ, UK \\ ' School of Applied Sciences, Bournemouth University, Poole, Dorset BH12 5BB, UK
}

\section{A R T I C L E I N F O}

\section{Article history:}

Received 24 October 2013

Received in revised form 9 February 2014

Accepted 11 March 2014

Available online 19 March 2014

\section{Keywords:}

Endogenous biotin binding

Streptavidin

Biotinylated secondary antibody

Qdot 625 streptavidin conjugate

Non-specific bands

Western blot

\begin{abstract}
A B S T R A C T
Endogenous biotin or biotinylated protein binding activity is a major drawback to biotin-avidin/ streptavidin detection system. The avidin/streptavidin conjugate used to detect the complex of the biotinylated secondary antibody and the primary antibody binds to endogenous biotin or biotinylated proteins leading to non-specific signals. In Western blot, the endogenous biotin or biotinylated protein binding activity is usually manifested in the form of $\sim 72 \mathrm{kDa}, \sim 75 \mathrm{kDa}$ and $\sim 150 \mathrm{kDa}$ protein bands, which often mask the signals of interest. To overcome this problem, a method based on prior hybridisation of the biotinylated secondary antibody and the streptavidin conjugate was developed. The method was tested alongside the conventional biotin-streptavidin method on proteins extracted from zebrafish (Danio rerio) embryos. Results showed that the newly developed method efficiently suppresses the endogenous biotin or biotinylated protein binding activity inherent to the biotin-streptavidin detection system.
\end{abstract}

(c) 2014 Elsevier B.V. All rights reserved.

\section{Introduction}

Biotin-avidin/streptavidin detection system is one of the most commonly used techniques for detecting an antigen of interest using immunological techniques such as Western blot, enzyme-linked immunosorbent assay, immunohistochemistry and immunocytochemistry. Biotin is present in all eukaryotic cells where it acts as a cofactor for carboxylase enzymes such as acetyl-CoA carboxylase (220 kDa), pyruvate carboxylases (130 kDa), propionyl-CoA carboxylase (75 kDa) and 3-methyl crotonyl-CoA carboxylases (72 kDa) (Vaitaitis et al., 1999; Banks et al., 2003; Lombard and Moreira, 2011). Consequently, techniques based on biotin-avidin/streptavidin detection system suffer from a major drawback. Avidin or streptavidin conjugated to a reporter molecule binds to the

\footnotetext{
* Corresponding author. Tel.: +44 1582 743700; fax: +44 1582743701. E-mail address: raju79@hotmail.co.uk (R. Ahmed).
}

endogenous biotin molecule in the biotin-dependent carboxylases regardless of the primary or the secondary antibody used, generating non-specific signals (Fig. 1). In Western blot, such non-specific signals result in extra protein bands corresponding to the size of the biotin-dependent carboxylases (Chandler and Ballard, 1985, 1986; Haneji and Koide, 1989; Praul et al., 1998; Vaitaitis et al., 1999; Banks et al., 2003). These often mask the protein bands of similar size making it impossible to interpret post translational protein modification such as phosphorylation (Banks et al., 2003; Yu et al., 2009).

To suppress the endogenous biotin binding activity in biotinavidin/streptavidin detection system, a second blocking step is incorporated into the method in question. In this blocking step, endogenous biotin or biotinylated protein is blocked using a tetrameric biotin binding protein such as avidin or streptavidin. The remaining biotin-binding sites on avidin or streptavidin molecule are then blocked using free biotin molecules (Wood and Warnke, 1981; Vaitaitis et al., 1999). However, avidin and 


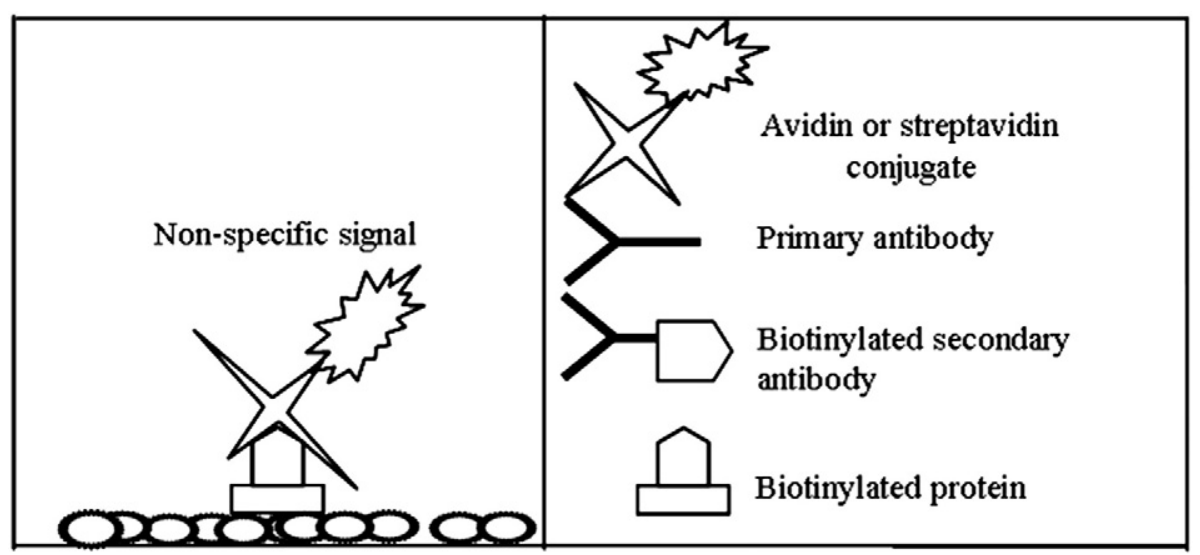

Fig. 1. Schematic diagram illustrating the endogenous biotin binding activity in biotin-avidin/streptavidin detection system. A non-specific signal is generated when the avidin/streptavidin conjugate interacts with the endogenous biotin or biotinylated proteins.

streptavidin are very costly proteins and, therefore, this blocking step can be very expensive depending on the extent of the endogenous biotin or biotinylated protein in the samples. In addition, the second blocking step is time consuming and it also compromises the quality of the specific signals by generating high backgrounds in the blot.

In this paper, we present a method based on prior hybridisation of the biotinylated secondary antibody with the streptavidin conjugate that efficiently suppresses the endogenous biotin binding activity in Western blot based on biotin-streptavidin detection system without the need for a separate blocking step. Prior hybridisation facilitates saturation of the biotin-binding sites in the streptavidin conjugate with the biotin molecule bound to the secondary antibody. The saturated streptavidin conjugate thus cannot bind to endogenous biotin or biotinylated proteins; and the interaction between the biotinylated secondary antibody bound to the streptavidin conjugate and the primary antibody bound to the antigen of interest is the only way a signal could be generated (Fig. 2). As the method described in this paper works by blocking the biotin binding sites in streptavidin conjugate rather than blocking the endogenously biotinylated proteins, it does not have any effect on the blot.

\section{Materials and method}

\subsection{Biotin, streptavidin reagents and antibodies}

Reagents were obtained from the following sources: Biotinylated goat anti-rabbit, Qdot 625 streptavidin conjugate, WesternDot blocking buffer, WesternDot wash buffer (WesternDot $^{\mathrm{TM}} 625$ Western Blot kits, W10142, Life Technologies), rabbit anti- $\beta$ actin (55339, Anaspec), MagicMark тм XP Western protein standards (LC5602, Life Technologies), SYPRO Ruby (S-11791, Life technologies).

2.2. Pre-hybridisation of the biotinylated goat anti-rabbit and the Qdot 625 streptavidin conjugate

$0.05 \mathrm{mg} / \mathrm{ml}$ of biotinylated goat anti-rabbit was added to $0.01 \mathrm{mg} / \mathrm{ml}$ of Qdot 625 streptavidin conjugate in a microcentrifuge tube, mixed with gentle pipetting, and incubated for $1 \mathrm{~h}$ at room temperature. $0.06 \mathrm{mg} / \mathrm{ml}$ of free biotin in wash buffer was added to the tube, mixed with gentle pipetting, and incubated for $30 \mathrm{~min}$ at room temperature to ensure any free biotin-binding sites in the Qdot 625 streptavidin conjugate were saturated. The complex of the biotinylated goat

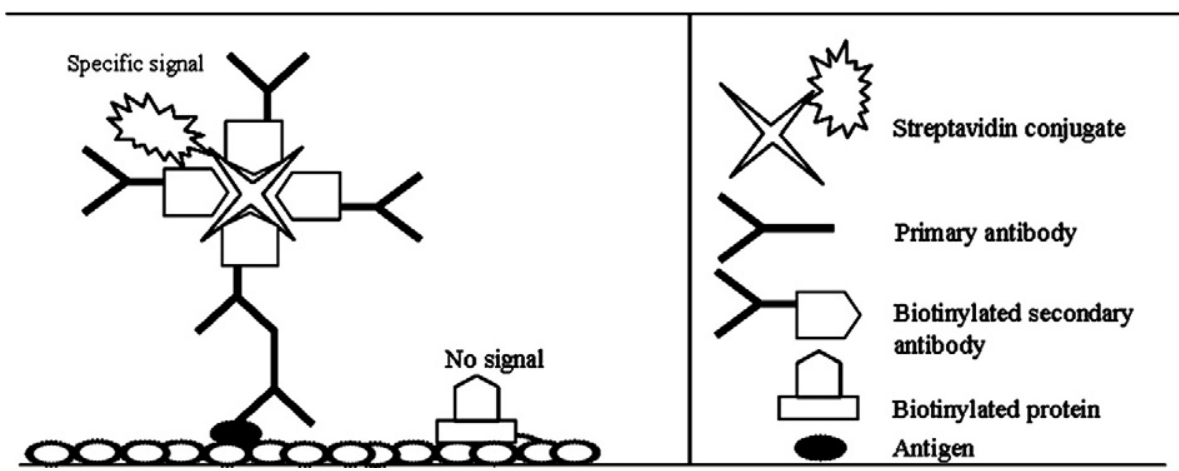

Fig. 2. Schematic diagram illustrating the pre-hybridisation method. Four biotin binding sites in streptavidin conjugate are blocked by the biotin molecule bound to the secondary antibody and therefore any signal generated would be the result of an interaction between the biotinylated secondary antibodies bound to the streptavidin conjugate and the primary antibody bound to the antigen of interest. 
anti-rabbit and the Qdot 625 streptavidin conjugate was then diluted in $10 \mathrm{ml}$ of WesternDot blocking buffer and stored at $4{ }^{\circ} \mathrm{C}$.

\subsection{Protein extraction, blotting and blocking}

The pre-hybridisation method is illustrated using zebrafish (Danio rerio) embryonic proteins. Total proteins from the embryos were extracted using the RIPA buffer according to the manufacturer's instruction (R0278, Sigma-Aldrich). All Western blot experiments described in this paper were carried out using $40 \mu \mathrm{g}$ of total proteins unless stated otherwise. Total proteins were resolved in sodium dodecyl sulphate polyacrylamide gel electrophoresis using Mini-Protean Tetra cell system (165-8000, Bio-Rad) according to the manufacturer's instruction. Gels were blotted onto PVDF membrane (88518, Thermo Scientific) for $45 \mathrm{~min}$ at $15 \mathrm{~V}$ using the semi-dry blotting method (170-3940, Bio-Rad). The blotted membranes were blocked with the WesternDot blocking buffer for $1 \mathrm{~h}$ at room temperature.

\subsection{Immunodetection}

\subsubsection{Conventional biotin-streptavidin method}

Following blocking, the membrane was incubated with $0.1 \mathrm{mg} / \mathrm{ml}$ of rabbit anti- $\beta$ actin in $10 \mathrm{ml}$ wash buffer overnight at $4{ }^{\circ} \mathrm{C}$, washed for $15 \mathrm{~min}$ with three changes of wash buffer, incubated with $0.01 \mathrm{mg} / \mathrm{ml}$ of goat anti-rabbit in $10 \mathrm{ml}$ wash buffer for $1 \mathrm{~h}$ at room temperature, washed for $15 \mathrm{~min}$ with three changes of wash buffer, incubated with $0.01 \mathrm{mg} / \mathrm{ml}$ of Qdot 625 streptavidin conjugate in 10-ml WesternDot blocking buffer for $1 \mathrm{~h}$ at room temperature, washed for $15 \mathrm{~min}$ with three changes of wash buffer followed by a final wash in $10 \mathrm{ml}$ Milli-Q water for five minutes.

\subsubsection{Probing with the streptavidin conjugate alone}

Following blocking, the membrane was incubated with $0.01 \mathrm{mg} / \mathrm{ml}$ of Qdot 625 streptavidin conjugate in $10 \mathrm{ml}$ WesternDot blocking buffer for $1 \mathrm{~h}$, washed for $15 \mathrm{~min}$ with three changes of wash buffer followed by a final wash in $10 \mathrm{ml}$ Milli-Q water for five minutes.

\subsubsection{SYPRO Ruby staining}

Following blocking, the membrane was incubated in $10 \mathrm{ml}$ solution of $7 \%$ acetic acid and $10 \%$ methanol for $10 \mathrm{~min}$ at room temperature, washed for 15 min with three changes of Milli-Q water. The membrane was then incubated in SYPRO Ruby stain for $15 \mathrm{~min}$ at room temperature, washed for three minutes with three changes of Milli- $\mathrm{Q}$ water.

\subsubsection{Probing with the pre-hybridised complex of the biotinylated secondary antibody and the streptavidin conjugate alone}

Following blocking, the membrane was incubated with the pre-hybridised complex of the biotinylated goat anti-rabbit and the Qdot 625 streptavidin conjugate in WesternDot blocking buffer for $1 \mathrm{~h}$, washed for $15 \mathrm{~min}$ with three changes of wash buffer followed by a final wash in Milli-Q water for five minutes.

\subsubsection{Immunodetection using the pre-hybridisation method}

The blocked membrane was incubated with $0.1 \mathrm{mg} / \mathrm{ml}$ of rabbit anti- $\beta$ actin in $10 \mathrm{ml}$ wash buffer overnight at $4{ }^{\circ} \mathrm{C}$, washed for $15 \mathrm{~min}$ with three changes of wash buffer, incubated with the pre-hybridised complex of the biotinylated goat anti-rabbit and the Qdot 625 streptavidin conjugate in WesternDot blocking buffer for $1 \mathrm{~h}$, washed for $15 \mathrm{~min}$ with three changes of wash buffer followed by a final wash in Milli-Q water for five minutes.

\subsubsection{Comparison of the conventional method and the pre-} hybridisation method

Two blots were prepared by resolving different amounts of total proteins ranging $10-50 \mu \mathrm{g}$ of total proteins. Following blocking, the membranes were incubated separately with $0.1 \mathrm{mg} / \mathrm{ml}$ of rabbit anti- $\beta$ actin in $10 \mathrm{ml}$ wash buffer overnight at $4{ }^{\circ} \mathrm{C}$, washed for $15 \mathrm{~min}$ with three changes of wash buffer. Then, one of the blots was developed using the conventional biotin-streptavidin detection system as described in Section 2.4.1, whereas, the second blot was developed using the pre-hybridisation method as described in Section 2.4.5.

\subsection{Visualisation}

Following the final wash in Milli-Q water, the membranes were visualised under UV light using a gel documentation system (82020-526, VWR) and images were taken.

\subsection{Densitometry and data analysis}

The density of $\beta$ actin bands across the samples were quantified using ImageJ software (Maryland, USA) according to the method described by Sheffield (2007).

\section{Results and discussion}

To demonstrate the extra protein bands generated from the endogenous biotin binding activity in Western blot based on biotin-streptavidin detection system, total proteins extracted from zebrafish (Danio rerio) embryos were probed with zebrafish specific anti- $\beta$ actin using the conventional method. The predicted molecular weight of zebrafish $\beta$ actin is $\sim 42 \mathrm{kDa}$ and the anti- $\beta$ actin detected a protein band of the expected size in the blot (Fig. 3A). However, there were also three very strong bands of approximately $\sim 72 \mathrm{kDa}, \sim 75 \mathrm{kDa}$ and $\sim 150 \mathrm{kDa}$ present in the blot. To demonstrate that those extra bands resulted from the interaction of the streptavidin conjugate with the endogenous biotin or biotinylated proteins, total proteins were probed with the Qdot 625 streptavidin conjugate alone in the absence of primary and secondary antibody. As expected, only the $\sim 72 \mathrm{kDa}, \sim 75 \mathrm{kDa}$ and $\sim 150 \mathrm{kDa}$ bands were detected in the blot (Fig. 3B). It is noteworthy here that the MagicMark ${ }^{\mathrm{TM}}$ XP Western protein standards used to estimate the size of the protein bands in this study consist of recombinant proteins with IgG binding sites; and the primary or the secondary antibody used for the detection of the antigen of interest bind to these IgG binding sites allowing direct visualisation of the protein standards in the blot. As the blot presented in Fig. 3B was developed in the absence of primary or secondary antibody; the protein standards were not visible in the blot. However, to 
A

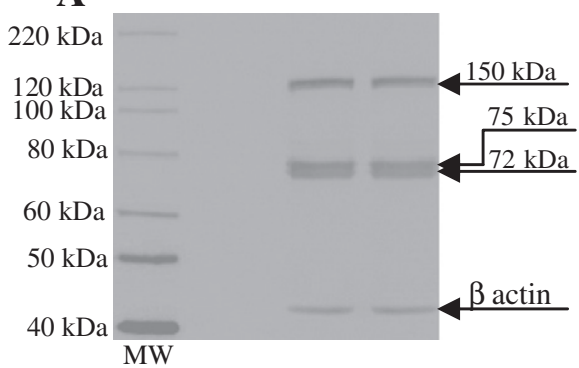

B

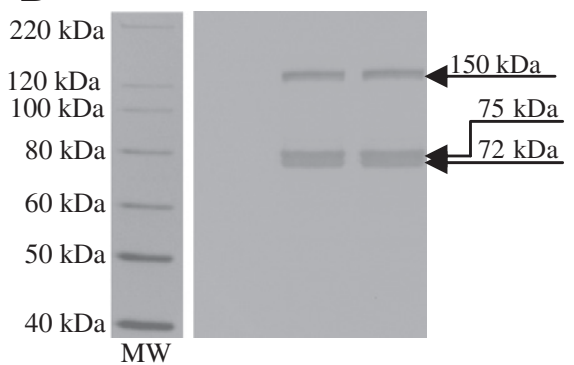

Fig. 3. Endogenous biotin or biotinylated protein binding activity in biotin-streptavidin detection system. (A) Showing the blot probed with anti- $\beta$ actin using the conventional biotin-streptavidin detection system. Three very intense bands at $\sim 72 \mathrm{kDa}, \sim 75 \mathrm{kDa}$ and $\sim 150 \mathrm{kDa}$, alongside the $\sim 42 \mathrm{kDa}$ band for $\beta$ actin, were detected in the membrane. (B) Showing the blot probed with the Qdot 625 streptavidin conjugate alone. Only the $\sim 72 \mathrm{kDa}, \sim 75 \mathrm{kDa}$ and $\sim 150 \mathrm{kDa}$ bands were detected in the membrane. MW represents MagicMark ${ }^{\mathrm{TM}}$ XP Western protein standards. MW shown in the blot in panel B was separately stained with SYPRO Ruby.

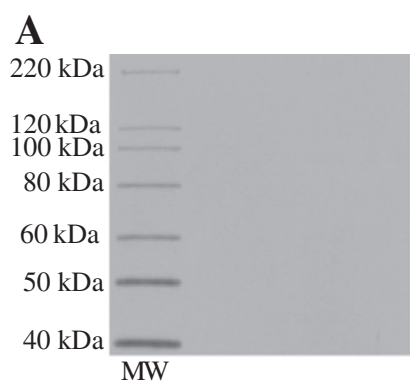

C

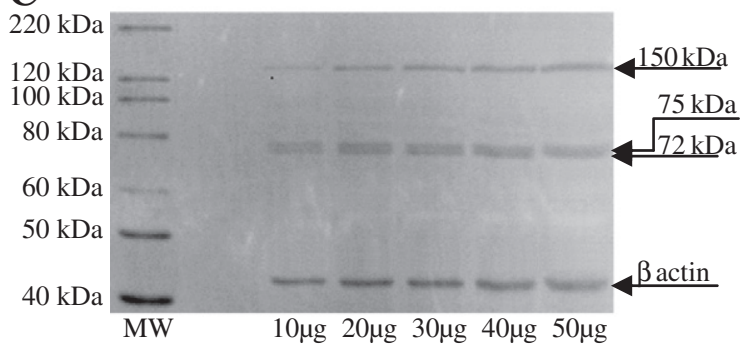

\section{B}

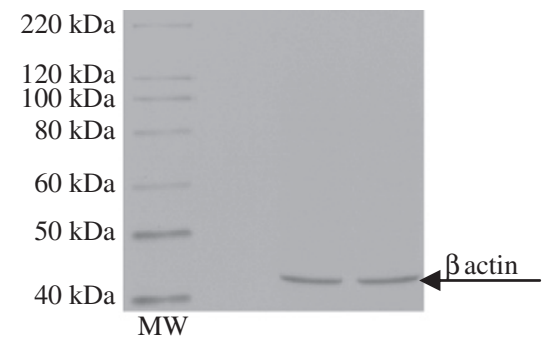

D

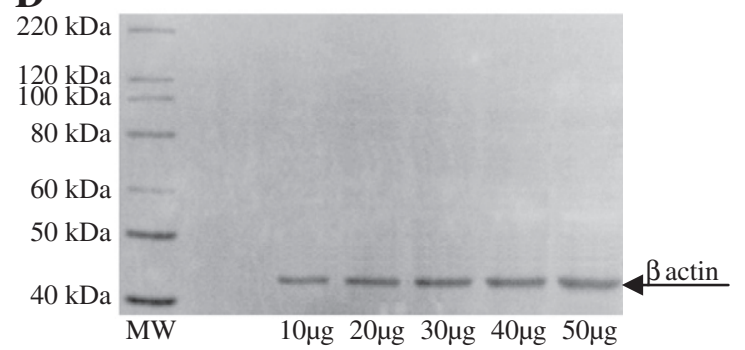

E

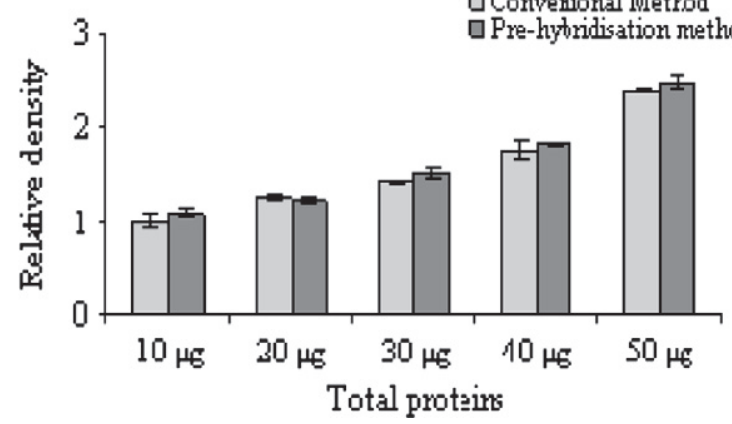

Fig. 4. Efficiency of the pre-hybridisation method to eliminate the endogenous biotin or biotinylated protein binding activity in biotin-streptavidin detection system. (A) Showing the blot probed with the pre-hybridised complex of the biotinylated goat anti-rabbit and the Qdot 625 streptavidin conjugate alone. The $\sim 72 \mathrm{kDa}, \sim 75 \mathrm{kDa}$ and $\sim 150 \mathrm{kDa}$ bands were not detected in the membrane. (B) Showing the blot probed with anti- $\beta$ actin using the pre-hybridisation method. Only the $\sim 42 \mathrm{kDa}$ band for $\beta$ actin was detected in the membrane. (C) Showing the blot probed for $\beta$ actin in a range of different amount of total proteins using the conventional biotin-streptavidin method. (D) Showing the blot probed for $\beta$ actin in a range of different amount of total proteins using the pre-hybridisation method. MW represents MagicMark ${ }^{\mathrm{TM}}$ XP Western protein standards. (E) Graphical presentation of the density of the bands for $\beta$ actin in a range of different amount of total proteins using the conventional biotin-streptavidin method and the pre-hybridisation method. Values shown are presented as relative to the density of $\beta$ actin band detected in $10 \mu \mathrm{g}$ lane in the blot developed using the conventional method. T-test showed that there was no statistically significant difference $(p>0.05)$ in the values compared. 
estimate the size of the protein bands, the protein standards in the blot in Fig. 3B were stained separately with a total protein stain, SYPRO Ruby. The findings indicate that the extra bands were indeed generated from the interaction of the Qdot 625 streptavidin conjugate with the endogenously biotinylated proteins and the $\sim 42 \mathrm{kDa}$ band detected in the previous blot (Fig. 3A) was indeed that of the structural protein $\beta$ actin.

The $\sim 72 \mathrm{kDa}$ and $\sim 75 \mathrm{kDa}$ bands detected in the blots are most likely to represent the biotin-dependent enzyme 3-methyl crotonyl-CoA carboxylase $(72 \mathrm{kDa})$ and propionyl-CoA carboxylase $(75 \mathrm{kDa})$, respectively. Although the predicted molecular weight of pyruvate carboxylases is $130 \mathrm{kDa}$, the enzyme has been reported to migrate at $\sim 150 \mathrm{kDa}$ in SDS-PAGE, probably due to post translational modification (Bayer et al., 1986; Yu et al., 2009; Lifespan Biosciences Inc, 2013; Sigma-Aldrich, 2013; Thermo Scientific, 2013). Therefore, the $\sim 150 \mathrm{kDa}$ band detected in the blot is likely to represent the biotin-dependent enzyme pyruvate carboxylase.

To demonstrate the efficiency of the pre-hybridisation method to suppress the endogenous biotin binding activity, total proteins were probed with the pre-hybridised complex of the biotinylated goat anti-rabbit and the Qdot 625 streptavidin conjugate alone in the absence of primary antibody. The result showed that the pre-hybridised complex did not generate the $\sim 72 \mathrm{kDa}, \sim 75 \mathrm{kDa}$ and $\sim 150 \mathrm{kDa}$ protein bands in the blot (Fig. 4A), indicating that the newly developed method successfully suppressed the endogenous biotin binding activity. To demonstrate the efficiency of the pre-hybridisation method to detect an antigen of interest, total proteins were probed with anti- $\beta$ actin using the pre-hybridised complex of the biotinylated goat anti-rabbit and the Qdot 625 streptavidin conjugate. The results showed that the pre-hybridisation method generated a single band of $\sim 42 \mathrm{kDa}$ for the structural protein, $\beta$ actin, in the blot (Fig. 4B). The finding indicates that the newly developed is very efficient in detecting an antigen of interest. Finally, a comparison of the sensitivity of the pre-hybridisation method with that of the conventional method over a range of antigen concentrations was carried out (Fig. 4C and D). The results showed that there was no significant difference $(p>0.05)$ in the density of the bands for $\beta$ actin as detected by both of the methods (Fig. 4E). The finding indicates that the prehybridisation method presented in this paper is as sensitive as the conventional biotin-streptavidin method.

\section{Conclusion}

In this paper we demonstrated that the pre-hybridisation method efficiently suppressed the endogenous biotin or biotinylated protein binding activity inherent to biotin-streptavidin detection system without compromising the signal for the antigen of interest. The method allows application of the biotinstreptavidin detection system to perform Western blot work with cells from zebrafish (Danio rerio) embryos and it will be applicable to tissues from kidney, liver and mammary gland, which are rich in endogenous biotin or biotinylated proteins (Dakshinamurti and Mistry, 1963; Moss and Lane, 2006; Taniguchi and Watanabe, 2007; Wood and Barden, 1977).
Although the method presented here was developed for application in Western blot, it may also be applied to other immunological techniques based on the same principle.

\section{Conflict of interest}

The authors declare no conflict of interest

\section{Acknowledgement}

This work was funded by the Overseas Research Students Awards Scheme (ORSAS), UK and the strategic research fund from the Institute of Biomedical and Environmental Sciences and Technology (iBEST), University of Bedfordshire, UK.

\section{References}

Banks, R.E., Craven, R.A., Harnden, P.A., Selby, P.J., 2003. Use of a sensitive EnVision ${ }^{\mathrm{TM}}+$-based detection system for Western blotting: avoidance of streptavidin binding to endogenous biotin and biotin-containing proteins in kidney and other tissues. Proteomics 3, 558.

Bayer, E.A., Ben-Hur, H., Gitlin, G., Wilchek, M., 1986. An improved method for the single-step purification of streptavidin. J. Biochem. Biophys. Methods 13, 103.

Chandler, C.S., Ballard, F.J., 1985. Distribution and degradation of biotincontaining carboxylases in human cell lines. Biochem. J. 232 (2), 385 (1985 Dec 1).

Chandler, C., Ballard, F., 1986. Multiple biotin-containing proteins in 3 T3-L1 cells. Biochem. J. 237, 123.

Dakshinamurti, K., Mistry, S.P., 1963. Tissue and intracellular distribution of biotin-C140OH in rats and chicks. J. Biol. Chem. 238, 294.

Haneji, T., Koide, S.S., 1989. Transblot identification of biotin-containing proteins in rat liver. Anal. Biochem. 177, 57.

Lombard, J., Moreira, D., 2011. Early evolution of the biotin-dependent carboxylase family. BMC Evol. Biol. 11, 232.

Lifespan Biosciences Inc., 2013. Anti-PC/pyruvate carboxylase antibody (internal) IHC-plus ${ }^{\mathrm{TM}}$ LS-B2457. Available online at www.lsbio.com/antibodies/anti-pcantibody-pyruvate-carboxylase-antibody-internal-goat-anti-humanpolyclonal-for-ihc-western-blot-ihc-plus-ls-b2457/84504 Accessed on $20 / 11 / 2012$.

Moss, J., Lane, M.D., 2006. The biotin-dependent enzymes. Advances in Enzymology and Related Areas of Molecular Biology. John Wiley \& Sons, Inc.

Praul, C.A., Brubaker, K.D., Leach, R.M., Gay, C.V., 1998. Detection of endogenous biotin-containing proteins in bone and cartilage cells with streptavidin systems. Biochem. Biophys. Res. Commun. 247, 312.

Sheffield, J., 2007. Imagej, a useful tool for biological image processing and analysis. Microsc. Microanal. 13 (SupplementS02), 200.

Sigma-Aldrich, 2013. Anti-pyruvate carboxylase antibody produced in goat. Available online at www.sigmaaldrich.com/catalog/product/sigma/ sab2500845 lang $=$ en\&region $=\mathrm{GB}$ (Accessed on 20/10/2012).

Taniguchi, A., Watanabe, T., 2007. Roles of biotin in growing ovarian follicles and embryonic development in domestic fowl. J. Nutr. Sci. Vitaminol. 53, 457.

Thermo Scientific, 2013. Anti-pyruvate carboxylase, polyclonal. Available online at www.thermoscientific.com/ecomm/servlet/productsdetail_ 11152_L10596_98464_14512039_-1 (Accessed on 20/11/2012).

Vaitaitis, G.M., Sanderson, R.J., Kimble, E.J., Elkins, N.D., Flores, S.C., 1999. Modification of enzyme-conjugated streptavidin-biotin Western blot technique to avoid detection of endogenous biotin-containing proteins. Biotechniques 26, 854

Wood, G., Warnke, R., 1981. Suppression of endogenous avidin-binding activity in tissues and its relevance to biotin-avidin detection systems. J. Histochem. Cytochem. 29, 1196.

Wood, H.G., Barden, R.E., 1977. Biotin enzymes. Annu. Rev. Biochem. 46, 385.

Yu, B., Qin, Z., Wijewickrama, G.T., Edirisinghe, P., Bolton, J.L., Thatcher, G.R.J., 2009. Comparative methods for analysis of protein covalent modification by electrophilic quinoids formed from xenobiotics. Bioconjug. Chem. 20, 728 